\title{
RF ACCELERATING STRUCTURES FOR THE MUON COOLING EXPERIMENT ${ }^{*}$
}

\author{
J.N. Corlett ${ }^{\#}$, D. Li, R.A. MacGill, M. Green, W.C. Turner, N. Hartman, LBNL, Berkeley, CA
}

N. Holtkamp, A. Moretti, FNAL, Batavia, IL

H. Kirk, R.B. Palmer, Y. Zhao, BNL, Brookhaven, NY

\begin{abstract}
\end{abstract}
D. Summers, Univ. of Mississippi, MS

beryllium windows through which the muon beams pass with low scattering rate [3,4]. This allows the shunt impedance of the device to be increased, the maximum surface field to equal the on-axis accelerating field, and the phase advance per cell to be arbitrarily chosen.

In addition to the pillbox design, we are studying a standing wave multicell cavity with open apertures, scaled to match the beam profile through the structure.

\section{RF SYSTEM REQUIREMENTS}

The purpose of the RF accelerating system is to replenish longitudinal momentum lost by the muons in scattering in the liquid hydrogen target. Each section must restore the longitudinal energy lost in the preceding target, resulting in an average gradient requirement of $34 \mathrm{MVm}^{-1}$ experienced by the muon beam.

Beryllium window thickness is limited by the additional emittance increase resulting from Coulomb scattering within the metal. Although the tolerance to $\mathrm{Be}$ is greater at the input to the cooling channel, where the emittance is largest, we choose to use the thinner $(127 \mu \mathrm{m})$ windows demanded at the low-emittance end of the cooling channel. An alternating solenoidal magnetic field will be applied throughout the cooling channel to focus the beam. Peak fields of $15 \mathrm{~T}$ or greater are produced by superconducting solenoids, and the RF structures must sit inside the bore of the solenoids. The length of the RF accelerating structure is $1.3 \mathrm{~m}$.

Table 1: Cooling section RF system specifications

An ionization cooling scheme has been developed, in which the muons lose momentum in a low- $\mathrm{Z}$ material, after which they regain their longitudinal momentum in an RF accelerating section. Sections of absorber and RF accelerator alternate inside a solenoidal magnetic focussing field, and bent solenoid emittance exchange sections provide 6-dimensional cooling. The entire cooling channel may consist of about 20 modules, with a factor 2 cooling per module.

The large transverse emittance of the muon beam requires large apertures, and the RF cavities must accommodate up to a $16 \mathrm{~cm}$ beam diameter. Such a large bore leads to a low shunt impedance in conventional open beam-pipe structures, however we propose to study cavities with up to $16 \mathrm{~cm}$ diameter thin $(127 \mu \mathrm{m})$

\footnotetext{
* *Work supported by the U.S. Department of Energy under Contract Nos. DE-AC0376SF00098 (LBNL), DE-AC02-76CH00016 (FNAL), DE-AC02-98CH10886 (BNL), DE-FG02-91ER40622 (University of Mississippi)

\# email: jncorlett@lbl.gov
} 
the end plates, through which the muon beam passes. The peak surface field is thus equal to the peak accelerating field on axis. Coupling between cells is through a sidecoupled cell, and the phase advance between cells is determined to be $\pi / 2$ by appropriate choice of cell length for the given muon velocity, and phase of drive to each chain of cells. Each chain is powered from a waveguide coupled into one of the accelerating cells. Coupling cells are coaxial structures, resonant at the frequency of the accelerating cells. The design has a high shunt impedance and is tolerant to dimensional errors.

To further increase the shunt impedance, we propose to investigate the possibility of cooling the structure to liquid nitrogen temperatures. The resulting increase in conductivity of copper is about a factor of two over room temperature, and a greater increase may be expected for the beryllium windows. The physical properties of beryllium at low temperatures, however, are not well determined and are the subject of several channels of investigation, including the manufacture of a low-power test cavity with interchangeable windows. We expect an improvement in Q-value, and hence shunt impedance, of a factor of two at $77 \mathrm{~K}$.

\subsection{Beryllium windows}

The inclusion of beryllium windows, allowing the accelerating field to equal the peak surface field, and isolation of cells in the interleaved structure, increase the shunt impedance of the structure over conventional designs. The trade-offs are with difficulties in mechanical construction and stability of the structure, power and voltage handling capabilities, and dark current generation. Wakefield effects of the thin beryllium foils may be advantageous.

As the cavity cools to liquid nitrogen temperatures, differential contraction between the beryllium window and the copper body of the cavity results in compressive stresses on the beryllium, causing it to distort. The resulting frequency change is unacceptable, and we are studying window frame designs of composite materials and pre-stressing the foil to overcome the compressive forces on the foils. Heating by RF currents in the foil causes expansion and additional distortions, complicated by the sensitivity of beryllium materials properties to temperature [5]. It is clear that the stresses induced by RF heating are beyond yield strength if operated at room temperature, we are investigating solutions at $77 \mathrm{~K}$.

Voltage handling capability is being studied in tests at the A0 facility at FNAL. Here, small (16mm diameter) samples of beryllium foils of thickness varying from 125 $\mu \mathrm{m}$ to $50 \mu \mathrm{m}$ are being tested at high field in a $1.5 \mathrm{GHz}$ RF gun structure. To date, no damage to foils has been observed up to the maximum achievable gradient of 35 $\mathrm{MVm}^{-1}$. Tests will continue at higher gradients in an effort to determine the damage level for the foils. Dark current may also be investigated in this apparatus.
Additional tests are planned for a pillbox cavity with beryllium surfaces on the end plates, operated at high power and in a magnetic field to study surface breakdown. Wakefield effects of a subrelativistic beam in a pillbox structure are under investigation.

We will also test a low-power cavity to investigate several of these aspects, as described in section 5 .

Properties of the $\pi / 2$ interleaved structure at room temperature are listed in table 2.

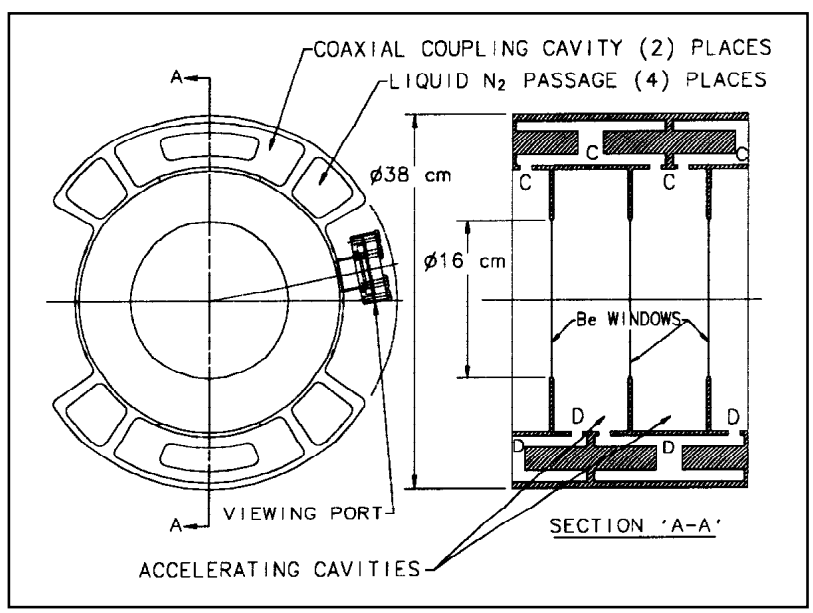

Figure 1: Layout of $\pi / 2$ interleaved cavity structure

Table 2: $\pi / 2$ interleaved cavity specifications (room temperature)

\begin{tabular}{|l|c|}
\hline Transit time corrected shunt impedance $\left(\mathrm{M} \Omega \mathrm{m}^{-1}\right)$ & 37.8 \\
\hline $\mathrm{Q}_{0}$ & 19600 \\
\hline Transit time $\mathrm{T}$ & 0.9 \\
\hline Filling time $\tau(\mu \mathrm{s})$ & 3.9 \\
\hline Cell length $(\mathrm{cm})$ & 8.1 \\
\hline Power for $34 \mathrm{MVm}^{-1}\left(\mathrm{MW} \mathrm{m}^{-1}\right)$ & 31 \\
\hline
\end{tabular}

\section{OPEN IRIS STRUCTURE}

An alternative to the $\pi / 2$ interleaved structure is the conventional multi-cell cavity with open irises through which the beam passes. This design avoids the complications of mounting and controlling distortions in thin metal foils, but involves the use of more power (the structure has a lower shunt impedance), and operating at higher peak surface fields. Table 3 lists the RF characteristics of the open-iris structure shown in figure 2, a $\pi$-mode eight cell linac (a half-structure is modeled here). We plan to build three-cell structures to high power test in strong magnetic fields to determine the viability of operating cavities under conditions required in the muon cooling experiment. Lacking the more complex engineering challenges of the $\pi / 2$ interleaved structure, the open-iris design may allow the most rapid development of a structure for the initial cooling tests. 
Table 3: Open iris cavity specifications

\begin{tabular}{|l|c|}
\hline $\begin{array}{l}\text { Transit time corrected } \\
\text { shunt impedance }\left(\mathrm{M} \Omega \mathrm{m}^{-1}\right)\end{array}$ & 17 \\
\hline $\mathrm{Q}_{\mathrm{o}}$ & 29500 \\
\hline Transit time $\mathrm{T}$ & 0.6 \\
\hline Filling time $\tau(\mu \mathrm{s})$ & 12 \\
\hline Cell length $(\mathrm{cm})$ & 16.2 \\
\hline Power for $34 \mathrm{MVm}^{-1}\left(\mathrm{MW} \mathrm{m}^{-1}\right)$ & 68 \\
\hline
\end{tabular}

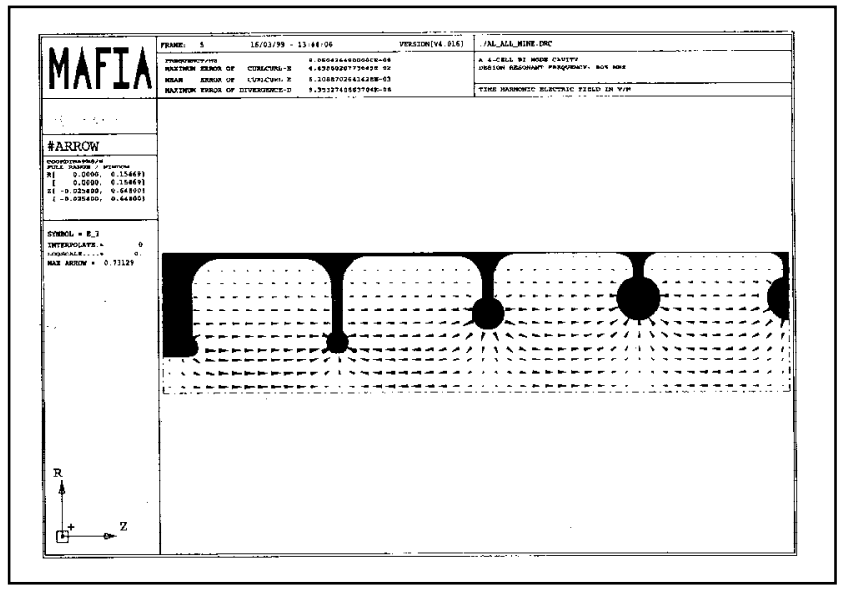

Figure 2: Open-iris cavity design, showing electric field in an 8-cell structure

\section{EXPERIMENTAL STATUS}

An experimental low-power test cavity consisting of two coupled half-cells and one full cell of the $\pi / 2$ interleaved cavity structure has been built to test mechanical stability, $\mathrm{Q}$, and thermal effects to LN2 temperatures. Figure 3 shows the two halves of the $\pi / 2$ interleaved cavity lowpower test structure. The irises are open in this view. The beryllium foils are diffusion bonded into in a sturdy beryllium ring, which is then clamped into the copper cavity with RF contact established by circumferential springs mounted inside the iris and clamp ring. Center conductors are mounted in the coupling cell, and the two halves and also end plates are bolted together. Springs mounted in recesses provide RF contact at joints, and tuning screws are provided for each cell along with coupling loops and probes for RF power input and measurements.

Cooling channels will be wrapped around the outside (grooves shown in figure 3), and the structure will be inserted into a vacuum tank with liquid nitrogen flowing through the cooling tubes to reduce it's operating temperature. Low-power measurements of the frequency and $\mathrm{Q}$ of modes will be made as a function of temperature. To test the effects of heating in the structure, a highpower feed will be provided also. An average power of up to $450 \mathrm{~W}$ is required to simulate the average power dissipation under long-pulse operation in the cooling channel RF systems.

The low-power cavity is being measured at LBNL, currently at room temperature, and measurements at low temperatures are scheduled to begin soon.

Beryllium properties are also being investigated in experimental apparatus at BNL, and at the University of Mississippi. Measurements of beryllium strips in a structure resonant at $805 \mathrm{MHz}$ have been made as a function of ambient temperature, to determine the resistivity of beryllium in commercial grade foils. D.C. measurements are also being made, and initial results show a discrepancy between the low-temperature measurements at d.c. and at $805 \mathrm{MHz}$, with the RF measurements indicating substantially higher resistivity.

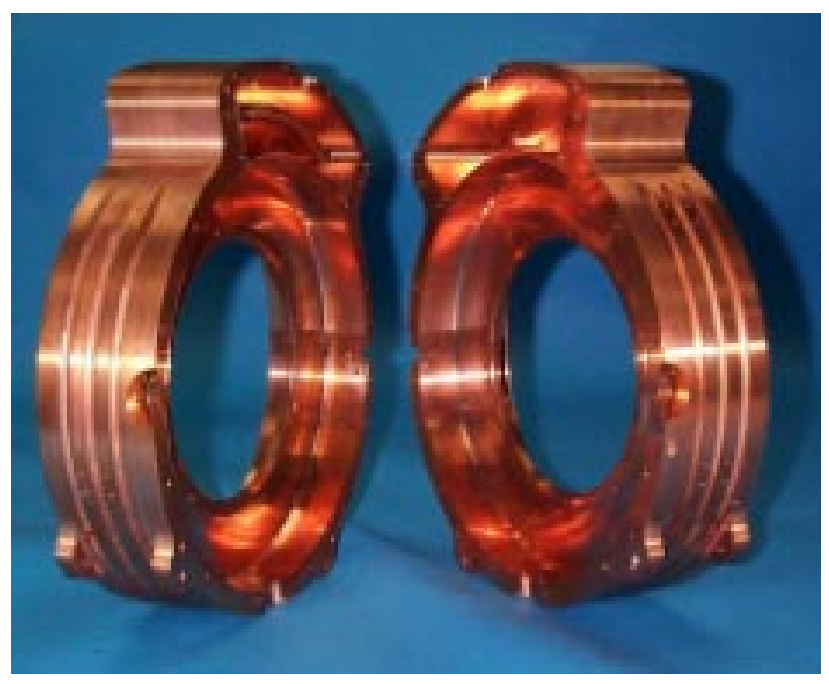

Figure 3: $\pi / 2$ interleaved cavity low-power test structure, copper cavity halves

High-power testing of cavities will take place at FNAL, within a 5T solenoidal magnetic field, and using an existing $805 \mathrm{MHz}$ klystron operating at up to $15 \mathrm{MW}$. Plans are well advanced, and high-power testing is scheduled to begin in September 1999.

\section{REFERENCES}

[1] R. Palmer, A. Tollestrup and A. Sessler, Proc. Of 1996 DPF, DPB Summer Study "New Directions for High Energy Physics", Snowmass, CO, (1996)

[2] "Status of Muon Collider Research and Development and Future Plans", Fermilab-PUB-98/179

[3] "RF System Concepts for a Muon Cooling Experiment", A. Moretti, J.N. Corlett, D. Li, W.C. Turner, H.G. Kirk, R.B. Palmer, Y. Zhao, European Particle Accelerator Conference, Stockholm, 1998.

[4] " $\pi / 2$ Interleaved Cavity Developments for the Muon Collider Cooling Experiment", A. Moretti, J.N. Corlett, D. Li, W.C. Turner, H.G. Kirk, R.B. Palmer, Y. Zhao, Linear Accelerator Conference, Chicago 1998.

[5] "Temperature Distribution Calculations on Beryllium Windows in RF cavities for a Muon Collider", D. Li, J. Corlett, W. Turner, Linear Accelerator Conference, Chicago 1998. 